\title{
SOME DENSE RANDOM PACKINGS GENERATED BY THE DEAD LEAVES MODEL
}

\author{
DOMINIQUE JEULIN \\ Mines ParisTech, PSL - Research University, Centre for Mathematical Morphology, 35 rue Saint-Honoré, \\ F-77300 Fontainebleau, France \\ e-mail: dominique.jeulin@mines-paristech.fr \\ (Received December 12, 2018; revised February 28, 2019; accepted February 28, 2019)
}

\begin{abstract}
The intact grains of the dead leaves model enables us to generate random media with non overlapping grains. Using the time non homogeneous sequential model with convex grains, theoretically very dense packings can be generated, up to a full covering of space. For these models, the theroretical volume fraction, the size distribution of grains, and the pair correlation function of centers of grains are given.
\end{abstract}

Keywords: dead leaves model, dense random packings.

\section{INTRODUCTION}

Packings of objects provide models for granular media. It is important for applications to get models showing a high volume fraction of grains, for instance for the prediction of macroscopic physical properties of random media. When using spheres with a fixed radius $R$, it is known that the highest volume fraction is 0.7404 , corresponding to Kepler's conjecture, which was proved by Th. Hales, as explained in Aste and Weaire (2000). A full covering of space can be obtained from the Apollonian packing (Aste and Weaire, 2000) involving a discrete size distribution of spheres. These ordered close packings provide regular tessellations of space in a deterministic way, which can be of interest to simulate crystal-like microstructures, but are not suitable to describe the variability observed in real media, which requires the use of probabilistic models.

In Delarue and Jeulin (2001), up to 0.55 volume fraction of spheres with the same radius could be obtained, starting from a dense CFC (cubic face centered) ordered arrangement of spheres, and combining random deletion and translations of spheres. Simulations of systems of non-overlapping long fibers (Altendorf and Jeulin, 2011) were developped from a combination of random walks and of a combination of attractive and repulsing forces.

We will follow here a different approach, based on a probabilistic model of non overlapping objects obtained by means of the dead leaves model (Matheron, 1968; Jeulin, 1980), when considering grains which remain uncovered during the sequence (Jeulin, 1998; 2000; 2002). When these grains are spherical with a fixed radius $R$, the centres of nonoverlapping spheres produce a standard Hard-Core point process (Matérn, 1960; Stoyan et al., 1987). Using the time homogeneous model provides random sets with a low volume fraction. In this paper, we show that dense packings can be obtained when using convex grains with a size distribution, and appropriate sequences during the generation of the model. In what follows, the results obtained for the time homogeneous case are reminded, and some sequential versions are studied, for which dense packing (surprisingly up to space filling) can be reached theoretically. Theoretical size distributions of grains generating these packings are provided. Finally the pair correlation function of centers of grains is given.

\section{INTACT GRAINS OF THE DEAD LEAVES MODEL FOR THE TIME HOMOGENEOUS CASE}

We first give a short reminder of basic definitions and results concerning the time homogeneous dead leaves tessellation of space (Matheron, 1968). We start from a sequence of random grains $A^{\prime}$ with Lebesgue measure $\mu_{n}\left(A^{\prime}\right)$ (or volume in the three-dimensionnal space) implanted in the $n$ dimensional space $\mathbb{R}^{n}$ according to a space-time point Poisson process with a constant intensity $\theta$. During the sequence, only parts of boundaries of new grains outside of already present grains are kept. By construction, the union of all grains appearing between time $u=0$ and time $u=t$ generates a standard Boolean model with primary grain $A^{\prime}$ and intensity $\theta t$. By analogy with dead leaves falling from trees, visible boundaries of grains of this version of the model are obtained by an observation of the tessellation from below (Jeulin, 1980; 1987; $1989 ; 1993 ; 1997)$. In a second step, only the intact 
grains are kept, to generate a random set $A(t)$ with nonoverlapping grains. The random set $A(t)$ is therefore obtained as the union of grains of the sequence falling outside of the earliest grains. The population of the intact grains in the dead leaves random tessellation can be used to estimate the characteristics of the random grains from an observation of the tessellation (Jeulin, 1993), or of its variants like primary functions in the case of the dead leaves random function (Jeulin, 1989; 1993; 1997).

We consider now the case where a single symmetric convex grain $A^{\prime}$ is used (for instance a sphere with radius $R$, or equivalently a cube, an ellipsoid, ...). The volume fraction (in $\mathbb{R}^{3}$ ) or more generally the probability $p(t)$ for a given point $x$ to belong to the random set $A(t)$ is given by

$$
\begin{aligned}
p(t) & =P\{x \in A(t)\} \\
& =\frac{1}{2^{n}}\left[1-\exp \left(-\theta t 2^{n} \mu_{n}\left(A^{\prime}\right)\right)\right] .
\end{aligned}
$$

For $t \rightarrow \infty$, we get $p=\frac{1}{2^{n}}$. As a result, the intact grains of a dead leaves model generate a random set of non overlapping grains with a limited packing density (namely $0.5,0.25$, and 0.125 in $\mathbb{R}, \mathbb{R}^{2}$ and $\mathbb{R}^{3}$ ).

Proof. The event " $x \in A(t)$ " can be written: at some $u<t, x$ is covered by a random grain $A^{\prime}$ falling outside of the previous occurences of grains $A^{\prime}$ from time 0 to time $u$ (generating a Boolean model with grain $A^{\prime}$ and with intensity $\theta u$ ). Therefore

$$
p(t)=\int_{0}^{t} \theta \mu_{n}\left(A^{\prime}\right) \exp \left(-\theta u 2^{n} \mu_{n}\left(A^{\prime}\right)\right) \mathrm{d} u
$$

since $\mu_{n}\left(A^{\prime} \oplus \check{A}^{\prime}\right)=2^{n} \mu_{n}\left(A^{\prime}\right)$ for any symmetric convex set $A^{\prime}$.

It turns out that replacing the single grain by a population of grains generates a random set with $p<$ $1 / 2^{n}$, as conjectured in Andersson et al. (2006) and shown in Kiderlen and Hörig (2000), so that the time homogeneous model with a single grain provides an upper bound of the probability $p$. It is the case of the two dimensional simulations given in Figs. 1 and 2 with area fraction 0.25 , obtained in the homogeneous case.This is rather low, as compared to the non random triangular close packing of discs, with area fraction $\pi / \sqrt{12}=0.9068$ (Aste and Weaire, 2000).

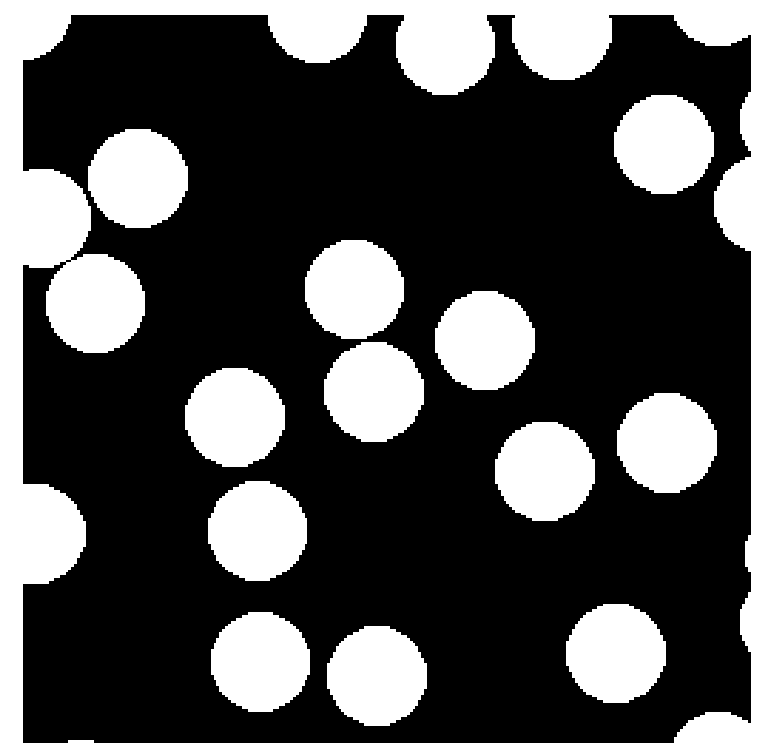

Fig. 1. Non overlapping discs built from the dead leaves model; area fraction 0.25 .

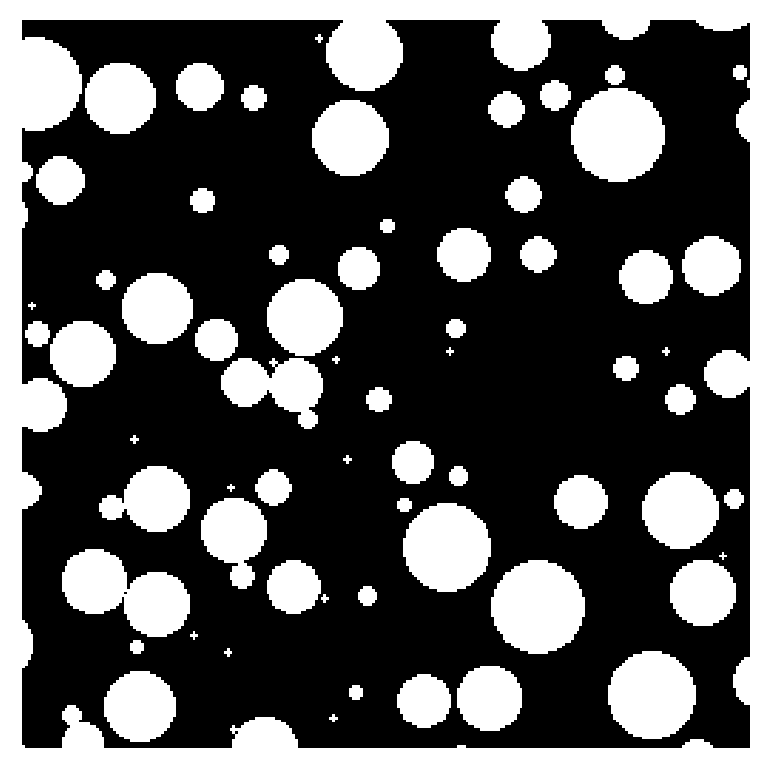

Fig. 2. Non overlapping discs with a size distribution, built from the dead leaves model; area fraction 0.25.

INTACT GRAINS OF THE DEAD LEAVES MODEL FOR THE GENERAL CASE

\section{SPACE FRACTION COVERED BY THE INTACT GRAINS}

We consider now a time sequence of independent random grains $A^{\prime}(t)$ implanted on Poisson points with intensity $\theta(t)$. Keeping the grains of the sequence falling outside of previous grains gives a random set $A(t)$. We have the following result. 
Theorem 1. The probability $p(t)$ is given by

$$
\begin{aligned}
p(t)= & P\{x \in A(t)\}=\int_{0}^{t} \theta(u) \mu_{n}\left(A^{\prime}(u)\right) \mathrm{d} u . \\
& \exp \left(-\int_{0}^{u} \theta(v) \mu_{n}\left(A^{\prime}(v) \oplus \check{A}^{\prime}(u)\right) \mathrm{d} v\right) .
\end{aligned}
$$

Proof. The event " $x \in A(t)$ " can be written: at some $u<t, x$ is covered by a random grain $A^{\prime}(u)$ falling outside of the previous occurences of grains $A^{\prime}(v)$ from time 0 to time $u$ (generating a sequential Boolean model with grain $A^{\prime}(v)$ and with intensity $\left.\theta(v)\right)$.

In what follows, we use a family of grains $A^{\prime}(t)$ with the same shape $A^{\prime}$ and with size $\lambda(t)$. When $A^{\prime}$ is a symmetric convex set,

$$
\mu_{n}\left(A^{\prime}(v) \oplus \check{A}^{\prime}(u)\right)=(\lambda(v)+\lambda(u))^{n} \mu_{n}\left(A^{\prime}\right),
$$

and $p(t)$ in Eq. 3 becomes:

$$
\begin{gathered}
p(t)=P\{x \in A(t)\}=\int_{0}^{t} \theta(u) \lambda^{n}(u) \mu_{n}\left(A^{\prime}\right) \mathrm{d} u . \\
\exp \left(-\int_{0}^{u} \theta(v) \mu_{n}\left(A^{\prime}\right)(\lambda(v)+\lambda(u))^{n} \mathrm{~d} v\right) .
\end{gathered}
$$

The aim of the present study is to find some combinations of functions $\theta(v)$ and $\lambda(v)$ providing large values of $p(t)$ derived from Equation (4), with possibly $p(t) \rightarrow 1$ for $t \rightarrow \infty$. For instance in the case of spherical grains, it is possible by this way to generate a space covering sequence of spheres (that might be so-called composite spheres, namely spheres of a first material embeded in a spherical shell made of a second material), as used for instance in physics and mechanics for the so-called Hashin composite spheres model (Hashin, 1962). This type of composite microstructure shows optimal physical properties such as macroscopic electrical conductivity, given the volume fraction and the individual properties of its two components. As far as we know, no theoretical study is available to generate such microstructures, despite its fundamental interest.

\section{SIZE DISTRIBUTION OF THE INTACT GRAINS}

It is possible to compute the "number" $f(\lambda)$ and the "measure" $g(\lambda)$ size distributions of the intact grains. For the "number" size distribution, every grain is counted with the same weight (1), whatever its size. For the "measure" size distribution, every grain is counted proportionally to its Lebesgue measure $\mu_{n}$, namely its volume in the three-dimensional space.
Using a decreasing sequence $\lambda(u)$, the size is univoquely parametrized by $u$. We give now the expressions of $f(\lambda(u))$ and of $g(\lambda(u))$.

Theorem 2. Provided the expression in the denominator below, giving the total number of grains per unit volume, is finite, the "number" size distribution $f(\lambda(u))$ is given by

$$
\begin{gathered}
f(\lambda(u))= \\
{\left[\theta(u) \exp \left(-\int_{0}^{u} \theta(v) \mu_{n}\left(A^{\prime}\right)(\lambda(v)+\lambda(u))^{n} \mathrm{~d} v\right)\right] /} \\
{\left[\int_{0}^{\infty} \theta(u) \mathrm{d} u \exp \left(-\int_{0}^{u} \theta(v) \mu_{n}\left(A^{\prime}\right)(\lambda(v)+\lambda(u))^{n} \mathrm{~d} v\right)\right] .}
\end{gathered}
$$

Proof. The specific number of intact grains per unit volume appearing between time $u$ and $u+d u$ is proportional to $\theta(u)$ and to the probability for the grain with size $\lambda(u)$ to be outside of the Boolean model made of all grains that appeared from time 0 to time $u$.

Theorem 3. The "measure" size distribution $g(\lambda(u))$ is given by

$$
\begin{aligned}
& g(\lambda(u))=\frac{1}{p(t)}\left[\theta(u) \lambda^{n}(u) \mu_{n}\left(A^{\prime}\right) .\right. \\
& \left.\exp \left(-\int_{0}^{u} \theta(v) \mu_{n}\left(A^{\prime}\right)(\lambda(v)+\lambda(u))^{n} \mathrm{~d} v\right)\right] .
\end{aligned}
$$

Proof. The probability $p_{i}(u)$ to belong to grains with size $\lambda(u)$ is derived from Eq. 4 :

$$
\begin{aligned}
p_{i}(u) & =\theta(u) \lambda^{n}(u) \mu_{n}\left(A^{\prime}\right) . \\
& \exp \left(-\int_{0}^{u} \theta(v) \mu_{n}\left(A^{\prime}\right)(\lambda(v)+\lambda(u))^{n} \mathrm{~d} v\right) .
\end{aligned}
$$

Normalizing $p_{i}(u)$ by the overall probability $p(t)$ gives Eq. 6.

\section{EXAMPLES OF MODELS OF DENSE PACKINGS}

Some specific cases, obtained for a particular choice of the functions $\theta(u)$ and $\lambda(u)$ used in the model, are detailed now. Of course other choices are possible and would also lead to dense packings. To generate a random set $A(t)$ with compact grains and to maximize $p(t)$, we decide to use an initial compact grain $A^{\prime}$ with measure $\mu_{n}\left(A^{\prime}\right)=1$ and a decreasing sequence $\lambda(v)$, with $\lambda(0)=1$. In what follows, numerical calculations and most 
analytical calculations were made with the software Mathematica.

From our results, packings obtained for an increasing function $\lambda(u)$ show a bounded volume fraction $p(t)<\frac{1}{2^{n}}$. Therefore to generate a packing with compact grains, decreasing functions $\lambda(u)$ are used, with $\lambda(0)=1$. To get a dense packing is considered the limit case obtained for $t \rightarrow \infty$. The union of all grains appearing between time 0 and time $t$ is a Boolean model $A_{1}(t)$ with $q_{1}(t)=1-$ $P\left\{x \in A_{1}(t)\right\}$. We have, noting $\lambda^{n}(u)=(\lambda(u))^{n}$

$$
q_{1}(t)=\exp \left(-\int_{0}^{t} \theta(u) \mu_{n}\left(A^{\prime}\right) \lambda^{n}(u) \mathrm{d} u\right) .
$$

Since by construction $p(t)=P\{x \in A(t)\}<1-q_{1}(t)$, we have to impose $q_{1}(\infty)=0$ to get the densest packings with $p(\infty)=p \simeq 1$. Therefore we will make a choice of functions $\theta(u)$ and $\lambda(u)$ satisfying

$$
\int_{0}^{\infty} \theta(u) \mu_{n}\left(A^{\prime}\right) \lambda^{n}(u) \mathrm{d} u=+\infty .
$$

In this paper, we make use of simple tractable analytical functions for $\theta(t)$ and $\lambda(t)$. Some other choices were made without success. A more general and systematic guideline would probably involve to work on variational techniques to maximize $p(t)$ in Eqs. 3 or 4, This is matter for further research, but the task is not easy, since the space of allowed functions $\theta(t)$ and $\lambda(t)$ is rather huge.

\section{CONSTANT RATE OF IMPLANTATION}

A constant rate of implantation (or time-space intensity) is obtained when $\theta(u) \mu_{n}\left(A^{\prime}\right) \lambda^{n}(u)=\theta$ (D. Jeulin, unpublished work). In that case, $q_{1}(t)=$ $\exp (-\theta t)$ and $q_{1}(t) \rightarrow 0$ when $t \rightarrow \infty$. With these assumptions, Eq. 4 becomes

$$
p(t)=\theta \int_{0}^{t} \mathrm{~d} u \exp \left(-\theta \int_{0}^{u}\left(1+\frac{\lambda(u)}{\lambda(v)}\right)^{n} \mathrm{~d} v\right),
$$

and the limit $p$ obtained for $p(t)$ when $t \rightarrow \infty$ is given by

$$
p=\theta \int_{0}^{\infty} \mathrm{d} u \exp \left(-\theta \int_{0}^{u}\left(1+\frac{\lambda(u)}{\lambda(v)}\right)^{n} \mathrm{~d} v\right) .
$$

For the model with a constant rate of implantation, the two size distributions become (dropping the denominator for $f(\lambda(u)))$ :

$$
\begin{aligned}
& f(\lambda(u)) \sim \\
& \quad \frac{\theta}{(\lambda(u))^{n} \mu_{n}\left(A^{\prime}\right)} \exp \left(-\theta \int_{0}^{u}\left(1+\frac{\lambda(u)}{\lambda(v)}\right)^{n} \mathrm{~d} v\right),
\end{aligned}
$$

$$
g(\lambda(u))=\frac{\theta}{p} \exp \left(-\theta \int_{0}^{u}\left(1+\frac{\lambda(u)}{\lambda(v)}\right)^{n} \mathrm{~d} v\right)
$$

For illustration, we consider some case studies in the three-dimensional case.

Case $1: \lambda(u)=1 /(1+u)^{\alpha}$

We get

$$
\int_{0}^{u}\left(1+\frac{\lambda(u)}{\lambda(v)}\right)^{3} \mathrm{~d} v=\int_{0}^{u}\left(1+\left(\frac{1+v}{1+u}\right)^{\alpha}\right)^{3} \mathrm{~d} v
$$

and

$$
\begin{aligned}
& \int_{0}^{u}\left(1+\left(\frac{1+v}{1+u}\right)^{\alpha}\right)^{3} \mathrm{~d} v= \\
& u+\frac{3(u+1)}{\alpha+1}+\frac{3(u+1)}{2 \alpha+1}+\frac{u+1}{3 \alpha+1} \\
&-\left(\frac{3}{2 \alpha+1}\left(\frac{1}{u+1}\right)^{2 \alpha}+\frac{1}{3 \alpha+1}\left(\frac{1}{u+1}\right)^{3 \alpha}\right) \\
&+\frac{3}{\alpha+1}\left(\frac{1}{u+1}\right)^{\alpha} .
\end{aligned}
$$

From numerical calculations, high concentrations can be obtained for different combinations of $\theta$ and $\alpha$. For instance, $p=0.909935$ for $\theta=1$ and $\alpha=100$; $p=0.949683$ for $\theta=0.1$ and $\alpha=100 ; p=0.953786$ for $\theta=0.01$ and $\alpha=100$. Higher values of $\theta$, in the range 1-10 produce lower volume fractions, decreasing with $\theta$.

To reach very high volume fractions, high coefficients $\alpha$ are required (typically $\alpha=100$ ), so that very small grains have to be generated during the simulation. Typically for $\theta=0.1$ and $\alpha=100$, about 150 orders of magnitude of sizes have to be generated with this set of parameters, which is out of reach in practical simulations.

We give in Table 1 the volume fraction reached for $\alpha=100$ and $\theta=1$ when stopping the simulations at time $t$ for which $\lambda(t)=10^{-2}, 10^{-3}$ and $10^{-6}$. This last range would correspond to grains in the scales between $1 \mathrm{~nm}$ and $1 \mathrm{~mm}$.

Table 1. Some examples of volume fractions for packings involving a finite range of sizes $(\alpha=100$ and $\theta=1)$.

\begin{tabular}{cccc}
\hline$\lambda(t)$ & $10^{-2}$ & $10^{-3}$ & $10^{-6}$ \\
\hline$p$ & 0.044 & 0.066 & 0.13116 \\
\hline
\end{tabular}


Case 2: $\lambda(u)=\exp (-\alpha u)$

When $\lambda(u)=\exp (-\alpha u)$,

$$
\int_{0}^{u}\left(1+\frac{\lambda(u)}{\lambda(v)}\right)^{3} \mathrm{~d} v=\int_{0}^{u}(1+\exp (-\alpha(u-v)))^{3} \mathrm{~d} v
$$

and

$$
\begin{aligned}
& \int_{0}^{u}(1+\exp (-\alpha(u-v)))^{3} \mathrm{~d} v= \\
& \frac{1}{6 \alpha}\left(29-2 \mathrm{e}^{-3 \alpha u}-9 \mathrm{e}^{-2 \alpha u}-18 \mathrm{e}^{-\alpha u}+6 \alpha u\right) .
\end{aligned}
$$

Very high values of $p$ are obtained for $\theta=0.01$ and $\alpha$ in the range $2-20$, where we get $0.975<p<$ 0.996. For $\alpha=1$ and $\theta=0.01, p=0.953185$. For $\alpha=5$ and $\theta=0.01, p=0.990395$. A full covering of space can be theroretically accessed with this model, but a covering fraction of 0.95 requires 131 orders of magnitude for the range of sizes involved in the simulation, which is non realistic in practice.

We give in Table 2 below the volume fraction reached for $\alpha=1$ and $\theta=0.01$ when stopping the simulations at time $t$ for which $\lambda(t)=10^{-2}, 10^{-3}$ and $10^{-6}$.

Table 2. Some examples of volume fractions for packings involving a finite range of sizes $(\alpha=1$ and $\theta=0.01)$.

\begin{tabular}{cccc}
\hline$\lambda(t)$ & $10^{-2}$ & $10^{-3}$ & $10^{-6}$ \\
\hline$p$ & 0.0435 & 0.064 & 0.1233 \\
\hline
\end{tabular}

Case 3: $\lambda(u)=\beta+1 /(1+u)^{\alpha}$

This variant of Case 1 enables us to generate populations of grains with a finite range of strictly positive sizes, since $\lambda(0)=\beta+1$ and $\lambda(\infty)=\beta$. When $\beta=0.1$, the typical range of sizes is around 10 , and when $\beta=0.01$, it is around 100 . In the present case, we have

$$
\begin{aligned}
\varphi(\beta, u) & =\int_{0}^{u}\left(1+\frac{\lambda(u)}{\lambda(v)}\right)^{3} \mathrm{~d} v \\
& =\int_{0}^{u}\left(1+\frac{\beta+(1+u)^{-\alpha}}{\beta+(1+v)^{-\alpha}}\right)^{3} \mathrm{~d} v .
\end{aligned}
$$

When $\alpha=1$, we get

$$
\begin{gathered}
\varphi(\beta, u)=\frac{1}{2 \beta^{4}(u+1)^{3}}\left(2 \beta u(2 \beta(u+1)+1)^{3}\right. \\
-6(\beta u+\beta+1)(2 \beta(u+1)+1)) \\
+\frac{1}{2 \beta^{4}(u+1)^{3}}(\beta u- \\
\left.6(\beta u+\beta+1)(2 \beta(u+1)+1)^{2} \log (\beta u+\beta+1)+\beta+1\right) \\
-\frac{(\beta u+\beta+1)}{2 \beta^{4}(u+1)^{3}}\left(\frac{(\beta u+\beta+1)^{2}}{(\beta+1)^{2}}\right. \\
-\frac{6(2 \beta(u+1)+1)(\beta u+\beta+1)}{\beta+1} \\
\left.-6(2 \beta(u+1)+1)^{2} \log (\beta+1)\right) .
\end{gathered}
$$

For illustration some calculations were made when $\alpha=1$. For $\beta=0.1$, a maximal value $p=0.198436$ is obtained for $\theta=0.05$. When $\beta=0.01$ a maximal value of $p=0.237714$ is given for $\theta=0.022$. When $\beta=0.001$, a maximal value of $p=0.256222$ is given for $\theta=0.007$. It seems that no dense packing can be reached with this version of the model.

When $\alpha=2$, we have

$$
\begin{aligned}
& \varphi(\beta, u)=\int_{0}^{u}\left(1+\frac{\beta+(1+u)^{-2}}{\beta+(1+v)^{-2}}\right)^{3} \mathrm{~d} v \\
& =-\frac{3}{8 \beta^{7 / 2}(u+1)^{6}}\left[25 \beta^{3}(u+1)^{6}+47 \beta^{2}(u+1)^{4}\right. \\
& \left.+27 \beta(u+1)^{2}+5\right] \tan ^{-1}(\sqrt{\beta}(u+1)) \\
& +\frac{8 \sqrt{\beta}(u+1)\left(2 \beta(u+1)^{2}+1\right)^{3}}{8 \beta^{7 / 2}(u+1)^{6}} \\
& -\frac{2 \sqrt{\beta}(u+1)\left(\beta(u+1)^{2}+1\right)}{8 \beta^{7 / 2}(u+1)^{6}} \\
& +\frac{3 \sqrt{\beta}(u+1)\left(\beta(u+1)^{2}+1\right)\left(7 \beta(u+1)^{2}+3\right)}{8 \beta^{7 / 2}(u+1)^{6}} \\
& +\frac{3}{8 \beta^{7 / 2}(u+1)^{6}}\left[25 \beta^{3}(u+1)^{6}+47 \beta^{2}(u+1)^{4}\right. \\
& \left.+27 \beta(u+1)^{2}+5\right] \tan ^{-1}(\sqrt{\beta}) \\
& -\frac{2 \sqrt{\beta}\left(\beta(u+1)^{2}+1\right)^{3}}{(\beta+1)^{2}\left(8 \beta^{7 / 2}(u+1)^{6}\right)} \\
& +\frac{3 \sqrt{\beta}\left(7 \beta(u+1)^{2}+3\right)\left(\beta(u+1)^{2}+1\right)^{2}}{(\beta+1)\left(8 \beta^{7 / 2}(u+1)^{6}\right)} \\
& +\frac{8 \sqrt{\beta}\left(2 \beta(u+1)^{2}+1\right)^{3}}{\left(8 \beta^{7 / 2}(u+1)^{6}\right)} .
\end{aligned}
$$


When $\beta=0.01$ a maximal value of $p=0.274566$ is given for $\theta=0.1$. When $\beta=0.001$, a maximal value of $p=0.315167$ is given for $\theta=0.05$.

No dense packing seems to be obtained with this limitation in the range of sizes.

Case 4: $\lambda(u)=\beta+\exp (-\alpha u)$

This variant of Case 2 generates again grains with a finite range of strictly positive sizes. We have

$$
\begin{gathered}
\int_{0}^{u}\left(1+\frac{\lambda(u)}{\lambda(v)}\right)^{3} \mathrm{~d} v=\int_{0}^{u}\left(1+\frac{\beta+\exp (-\alpha u)}{\beta+\exp (-\alpha v)}\right)^{3} \mathrm{~d} v \\
=\frac{e^{-3 \alpha u}}{2 \alpha \beta^{3}}\left(\beta^{3} e^{3 \alpha u}(16 \alpha u-9)+\beta^{2} e^{2 \alpha u}(24 \alpha u-11)\right. \\
-\frac{\left(\beta e^{\alpha u}+1\right)}{(\beta+1)^{2}}\left(2(\beta+1)^{2} \log (\beta+1) \cdot\right. \\
\left.\left(7 \beta^{2} e^{2 \alpha u}+5 \beta e^{\alpha u}+1\right)\right) \\
+\frac{\beta\left(\beta e^{\alpha u}+1\right)}{(\beta+1)^{2}}\left(9 \beta^{3} e^{2 \alpha u}+4 \beta^{2} e^{\alpha u}\left(2 e^{\alpha u}+3\right)\right. \\
\left.+\beta\left(10 e^{\alpha u}+3\right)+2\right) \\
+2\left(7 \beta^{3} e^{3 \alpha u}+12 \beta^{2} e^{2 \alpha u}+6 \beta e^{\alpha u}+1\right) \log \left(e^{-\alpha u}+\beta\right) \\
\left.+2 \beta e^{\alpha u}(6 \alpha u-1)+2 \alpha u\right)
\end{gathered}
$$

For $\alpha=1$ and $\beta=0.01$, a maximum of $p=$ 0.313331 is obtained for $\theta=0.15$. When $\beta=0.001$ (involving a range of 1000 in the sizes), a maximum of $p=0.379635$ is obtained for $\theta=0.13$. When $\beta=$ $10^{-4}, 10^{-5}$ or $10^{-6}$, a maximum of $p=0.431963$, $0.4735995,0.459862$ is obtained for $\theta=0.11,0.11$, 0.09 . Again no highly dense packing is accessed with this range of sizes and this model.

\section{EXAMPLES OF TIME DEPENDING RATES OF IMPLANTATION}

We will consider various cases where $\theta(u)$ and $\lambda(u)$ are respectively an increasing and a decreasing function of $u$, with the condition $\int_{0}^{\infty} \theta(u) \mu_{n}\left(A^{\prime}\right) \lambda^{n}(u) d u=+\infty$.

\section{Some polynomial laws}

When $\theta(u) \mu_{n}\left(A^{\prime}\right)=\theta(1+u)^{\beta}$ and $\lambda(u)=1 /(1+$ $u)^{\alpha}$, it comes

$$
\lambda^{n}(u) \theta(u) \mu_{n}\left(A^{\prime}\right)=\theta \frac{(1+u)^{\beta}}{(1+u)^{n \alpha}},
$$

and

$$
\begin{gathered}
\int_{0}^{u}(1+v)^{\beta}\left((1+v)^{-\alpha}+(1+u)^{-\alpha}\right)^{3} \mathrm{~d} v \\
=\left(2 \left(-3 \alpha^{3}+20 \alpha^{2}(\beta+1)-18 \alpha(\beta+1)^{2}\right.\right. \\
\left.\left.+4(\beta+1)^{3}\right)(u+1)^{-3 \alpha+\beta+1}\right) / \\
((\beta+1)(-3 \alpha+\beta+1)(-2 \alpha+\beta+1)(-\alpha+\beta+1)) \\
-\frac{(u+1)^{-3 \alpha}}{\beta+1}-\frac{3(u+1)^{-2 \alpha}}{-\alpha+\beta+1} \\
-\frac{3(u+1)^{-\alpha}}{-2 \alpha+\beta+1}-\frac{1}{-3 \alpha+\beta+1} .
\end{gathered}
$$

To get dense packings, we require $\beta \geq n \alpha-1$. Some typical results for $n=3$ are given in Table 3 .

Table 3. Probability p for some sets of parameters.

\begin{tabular}{llll}
\hline$\theta$ & 0.1 & 0.01 & 0.001 \\
\hline$\alpha=2, \beta=6$ & 0.317 & 0.358 & 0.364 \\
$\alpha=2, \beta=5.5$ & 0.414 & 0.478 & \\
\hline
\end{tabular}

A special case is given for $\beta=n \alpha-1$ and therefore $\beta=3 \alpha-1$. We have

$$
\begin{aligned}
& f(\alpha, u) \\
& =\int_{0}^{u}(1+v)^{3 \alpha-1}\left((1+v)^{-\alpha}+(1+u)^{-\alpha}\right)^{3} \mathrm{~d} v \\
& \quad=\frac{1}{2 \alpha}\left(-(u+1)^{-3 \alpha}-6(u+1)^{-2 \alpha}\right. \\
& \left.\quad+(u+1)^{-\alpha}(6 \alpha \log (u+1)+5)+2\right),
\end{aligned}
$$

and

$$
p=\theta \int_{0}^{\infty} \frac{\mathrm{d} u}{1+u} \exp (-\theta f(\alpha, u))
$$

For $\alpha=1$ and $\theta$ increasing from 0.01 to $0.1, p$ decreases from 0.95 to 0.6 . When $\theta$ increases from 0.001 to $0.01, p$ decreases from 0.995 to 0.95 .

For $\alpha=3, p$ decreases from 0.999 to 0.985 when $\theta$ increases from 0.001 to 0.01 .

For $\alpha=5, p$ decreases from 0.9999 to 0.9915 when $\theta$ increases from 0.001 to 0.01 .

Therefore this special case gives access to extremely high density of packings. As for the previous exponential case, 131 orders of magnitude for the range of sizes are required for a simulation where $p$ reaches 0.95 when $\alpha=1$ and $\theta=0.01$, which is practically out of reach.

We give in Table 4 below the volume fraction reached for $\alpha=1$ and $\theta=0.01$ when stopping 
the simulations at time $t$ for which $\lambda(t)=$ $10^{-2}, 10^{-3}$ and $10^{-6}$ and $10^{-6}$. They are close to what was obtained for cases 1 and 2 above.

Table 4. Some examples of volume fractions for packings involving a finite range of sizes $(\alpha=1$ and $\theta=0.01$ ).

\begin{tabular}{cccc}
\hline$\lambda(t)$ & $10^{-2}$ & $10^{-3}$ & $10^{-6}$ \\
\hline$p$ & 0.04325 & 0.064 & 0.1233 \\
\hline
\end{tabular}

\section{Use of exponential laws for $\lambda(u)$ and $\theta(u)$}

When $\lambda(u)=\mathrm{e}^{-\alpha u}$ and $\theta(u) \mu_{n}\left(A^{\prime}\right)=\theta \mathrm{e}^{\beta u}$, we require $\beta \geq n \alpha$ in order to obtain dense packings. In this case, we have

$$
\begin{aligned}
p=\theta \int_{0}^{\infty} \exp (\beta-3 \alpha) u \mathrm{~d} u & \\
& \quad \exp \left(-\theta \int_{0}^{u} \mathrm{e}^{\beta v}\left(\mathrm{e}^{-\alpha u}+\mathrm{e}^{-\alpha v}\right)^{3} \mathrm{~d} v\right)
\end{aligned}
$$

and (for $\beta \neq 3 \alpha, \beta \neq 2 \alpha, \beta \neq \alpha$ )

$$
\begin{array}{r}
\int_{0}^{u} \mathrm{e}^{\beta v}\left(\mathrm{e}^{-\alpha u}+\mathrm{e}^{-\alpha v}\right)^{3} \mathrm{~d} v \\
=\frac{\left(6 \alpha^{3}-40 \alpha^{2} \beta+36 \alpha \beta^{2}-8 \beta^{3}\right) \mathrm{e}^{u(\beta-3 \alpha)}}{\beta(\alpha-\beta)(2 \alpha-\beta)(3 \alpha-\beta)} \\
-\frac{\left(\mathrm{e}^{-\alpha u}+1\right)^{3}\left(-\frac{3 \mathrm{e}^{2 \alpha u}}{2 \alpha-\beta}-\frac{\mathrm{e}^{3 \alpha u}}{3 \alpha-\beta}-\frac{3 \mathrm{e}^{\alpha u}}{\alpha-\beta}+\frac{1}{\beta}\right)}{\left(\mathrm{e}^{\alpha u}+1\right)^{3}} .
\end{array}
$$

Typical results for $p$ are given in Table 5, where $\theta=0.01$. It appears that very dense packings are reached when $\beta \approx n \alpha$.

Table 5. Probability p for some sets of parameters in the case of exponential laws.

\begin{tabular}{rll}
\hline$\alpha$ & $\beta$ & $p$ \\
\hline 3 & 10 & 0.433 \\
5 & 16 & 0.54 \\
5 & 20 & 0.266 \\
5 & 15.1 & 0.904486 \\
7 & 21.1 & 0.929732 \\
7 & 25 & 0.34 \\
10 & 30.01 & 0.99043 \\
\hline
\end{tabular}

For illustration, the probability $p=0.93$ is reached for $\alpha=7$ and $\beta=21.1$, which requires 99 orders of magnitude in the involved range of sizes, and therefore cannot be implemented in simulations.

\section{Combination of exponential law and of polynomial law}

Using $\lambda(u)=1 /(1+u)^{\alpha}$ and $\theta(u)=\theta \mathrm{e}^{\beta u}$, we satisfy $q(\infty)=0$. We have

$$
\varphi(\alpha, \beta, u)=\int_{0}^{u} \mathrm{e}^{\beta v}\left((1+v)^{-\alpha}+(1+u)^{-\alpha}\right)^{3} \mathrm{~d} v .
$$

With this combination, we could not obtain high packing densities.

\section{SIZE DISTRIBUTIONS FOR DENSE PACKINGS}

It is interesting to derive theoretical size distributions (Eqs. 6 and 5) of grains involved in some cases of previously introduced dense packings.

\section{CONSTANT RATE OF IMPLANTATION \\ AND $\lambda(u)=1 /(1+u)^{\alpha}$}

The "measure" size distribution is derived from Eq. 7, replacing $u$ by its expression in $\lambda$. It is given by

$$
\begin{aligned}
g(\lambda) & =\frac{\theta}{p} \exp (-\theta[-1 \\
+\lambda^{-\alpha} & \left(1+\frac{3}{\alpha+1}+\frac{3}{2 \alpha+1}+\frac{1}{3 \alpha+1}\right) \\
& \left.\left.\quad\left(\frac{3 \lambda}{\alpha+1}+\frac{3 \lambda^{2}}{2 \alpha+1}+\frac{\lambda^{3}}{3 \alpha+1}\right)\right]\right),
\end{aligned}
$$

for $0 \leq \lambda \leq 1$.

We have $g(0)=0$ and $g(1)=\theta / p$. The pdf $g(\lambda)$ increases with $\lambda$.

The "number" size distribution is proportional to

$$
\begin{aligned}
f(\lambda) & \sim \frac{\theta}{p \mu_{n}\left(A^{\prime}\right) \lambda^{3}} \exp (-\theta[-1 \\
+\lambda^{-\alpha} & \left(1+\frac{3}{\alpha+1}+\frac{3}{2 \alpha+1}+\frac{1}{3 \alpha+1}\right) \\
& \left.\left.\quad-\left(\frac{3 \lambda}{\alpha+1}+\frac{3 \lambda^{2}}{2 \alpha+1}+\frac{\lambda^{3}}{3 \alpha+1}\right)\right]\right),
\end{aligned}
$$

for $0 \leq \lambda \leq 1$.

We have $f(1) \sim \frac{\theta}{p \mu_{n}\left(A^{\prime}\right)}$, and $f(\lambda) \rightarrow \infty$ for $\lambda \rightarrow 0$. 


\section{CONSTANT RATE OF IMPLANTATION}

AND $\lambda(u)=\exp (-\alpha u)$

The "measure" size distribution is derived from Eq. 8 , replacing $u$ by its expression in $\lambda$. It is given by

$$
g(\lambda)=\frac{\theta}{p} \lambda^{\frac{\theta}{\alpha}} \exp \left[-\frac{\theta}{6 \alpha}\left(29-18 \lambda-9 \lambda^{2}-2 \lambda^{3}\right)\right]
$$

for $0 \leq \lambda \leq 1$.

We have $g(1)=\frac{\theta}{p}$ and $g(0)=0$. The pdf $g(\lambda)$ increases with $\lambda$. This is illustrated in Fig. 3 for some sets of parameters.

The "number" size distribution is proportional to

$$
\begin{aligned}
& f(\lambda) \sim \\
& \quad \frac{\theta \lambda^{\theta / \alpha}}{p \mu_{n}\left(A^{\prime}\right) \lambda^{3}} \exp \left[-\frac{\theta}{6 \alpha}\left(29-18 \lambda-9 \lambda^{2}-2 \lambda^{3}\right)\right]
\end{aligned}
$$

for $0 \leq \lambda \leq 1$.

We have $f(1) \sim \frac{\theta}{p \mu_{n}\left(A^{\prime}\right)}$. If $\frac{\theta}{\alpha}<3, f(\lambda) \rightarrow \infty$ when $\lambda \rightarrow 0$. If $\frac{\theta}{\alpha}>3, f(\lambda) \rightarrow 0$ when $\lambda \rightarrow 0$. If $\theta=3 \alpha$, $f(0) \sim \frac{\theta}{p \mu_{n}\left(A^{\prime}\right)} \exp \left(-\frac{29}{2}\right)$.

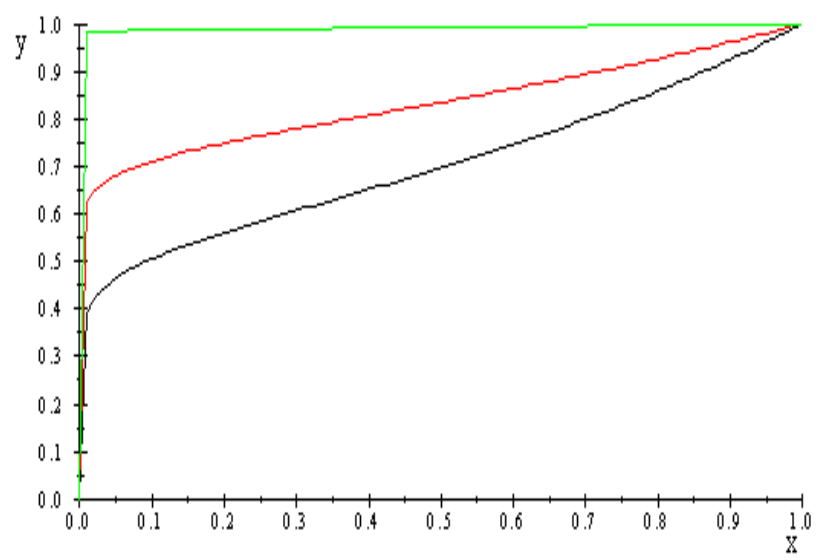

Fig. 3. $\frac{p}{\theta} g(\lambda)$ as a function of $\lambda$, for $\theta=0.1$ and $\alpha=1$ (black), $\theta=0.1$ and $\alpha=2$ (red), $\theta=0.01$ and $\alpha=5$ (green).

\section{TIME DEPENDING RATE OF IMPLANTATION AND POLYNOMIAL LAW}

We consider now the case when $\theta(u) \mu_{n}\left(A^{\prime}\right)=$ $\theta(1+u)^{\beta}$ and $\lambda(u)=1 /(1+u)^{\alpha}$, with $\beta=3 \alpha-1$. We get the "measure" size distribution, using Eqs. 6 and 9 ,

$$
\begin{array}{r}
g(\lambda)=\frac{\theta}{p} \lambda^{\frac{3 \theta \lambda+1}{\alpha}} \exp \left[-\frac{\theta}{2 \alpha}\left(2+5 \lambda-6 \lambda^{2}-\lambda^{3}\right)\right] \\
\text { for } 0 \leq \lambda \leq 1,
\end{array}
$$

with $g(1)=\theta / p$ and $g(0)=0$. The pdf $g(\lambda)$ increases with $\lambda$. It is illustrated in Fig. 4 for various sets of parameters. They are very different from what was obtained in Fig. 1.

The "number" size distribution is proportional to

$$
\begin{aligned}
& f(\lambda) \sim \\
& \frac{\theta}{p \mu_{n}\left(A^{\prime}\right) \lambda^{3}} \lambda^{\frac{3 \theta \lambda+1}{\alpha}} \exp \left[-\frac{\theta}{2 \alpha}\left(2+5 \lambda-6 \lambda^{2}-\lambda^{3}\right)\right],
\end{aligned}
$$

for $0 \leq \lambda \leq 1$.

We have $f(1) \sim \frac{\theta}{p \mu_{n}\left(A^{\prime}\right)}$. If $\alpha>1 / 3, f(\lambda) \rightarrow \infty$ when $\lambda \rightarrow 0$. If $\alpha<1 / 3, f(\lambda) \rightarrow 0$ when $\lambda \rightarrow 0$. If $\alpha=1 / 3, f(0) \sim \frac{\theta}{p \mu_{n}\left(A^{\prime}\right)} \exp (-3 \theta)$.

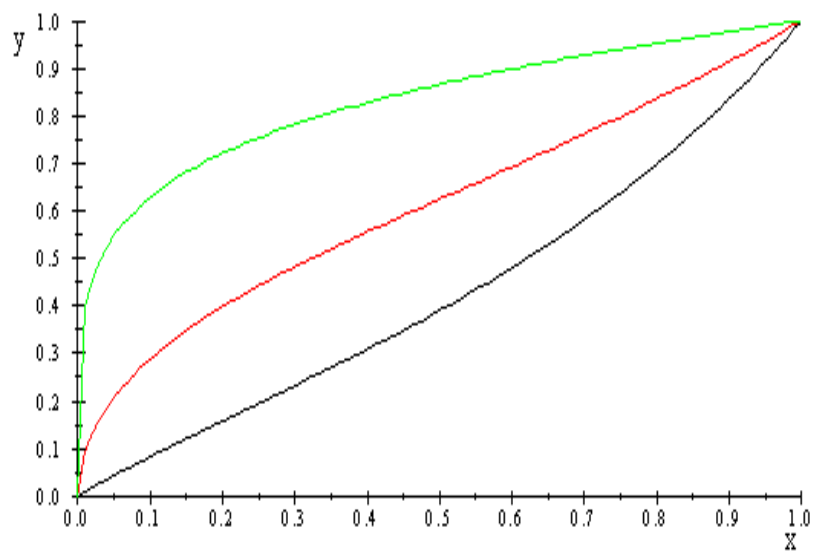

Fig. 4. $\frac{p}{\theta} g(\lambda) v$ as a function of $\lambda$, for $\theta=0.1$ and $\alpha=1$ (black), $\theta=0.1$ and $\alpha=2$ (red), $\theta=0.01$ and $\alpha=5$ (green).

\section{TIME DEPENDING RATE OF IMPLANTATION AND EXPONENTIAL LAW}

When $\lambda(u)=\exp (-\alpha u)$ and $\theta(u) \mu_{n}\left(A^{\prime}\right)=$ $\theta \exp (\beta u)$, the "measure" size distribution is obtained from Eqs. 6 and 10:

$$
\begin{aligned}
& g(\lambda)=\frac{\theta}{p} \lambda^{3-\beta / \alpha} \\
& \exp \left\{-\theta\left[\lambda^{3-\beta / \alpha} \frac{\left(6 \alpha^{3}-40 \alpha^{2} \beta+36 \alpha \beta^{2}-8 \beta^{3}\right)}{\beta(\alpha-\beta)(2 \alpha-\beta)(3 \alpha-\beta)}\right.\right. \\
&\left.\left.\quad-\left(-\frac{1}{3 \alpha-\beta}-\frac{3 \lambda}{2 \alpha-\beta}-\frac{3 \lambda^{2}}{\alpha-\beta}+\frac{\lambda^{3}}{\beta}\right)\right]\right\}
\end{aligned}
$$

for $0 \leq \lambda \leq 1$; with with $g(1)=\theta / p$ and $g(0)=0$. 
The "number" size distribution is proportional to

$$
\begin{aligned}
& f(\lambda) \sim \frac{\theta}{p \mu_{n}\left(A^{\prime}\right)} \lambda^{-\beta / \alpha} \\
& \exp \left\{-\theta\left[\lambda^{3-\beta / \alpha} \frac{\left(6 \alpha^{3}-40 \alpha^{2} \beta+36 \alpha \beta^{2}-8 \beta^{3}\right)}{\beta(\alpha-\beta)(2 \alpha-\beta)(3 \alpha-\beta)}\right.\right. \\
&\left.\left.\quad-\left(-\frac{1}{3 \alpha-\beta}-\frac{3 \lambda}{2 \alpha-\beta}-\frac{3 \lambda^{2}}{\alpha-\beta}+\frac{\lambda^{3}}{\beta}\right)\right]\right\}
\end{aligned}
$$

for $0 \leq \lambda \leq 1$. $\lambda \rightarrow 0$.

We have $f(1) \sim \theta /\left(p \mu_{n}\left(A^{\prime}\right)\right)$ and $f(\lambda) \rightarrow \infty$ when

\section{PAIR CORRELATION FUNCTION OF INTACT GRAINS CENTERS}

For a point process we can define the pair correlation function $G(r)$ as a function of the density $\rho$ (average number of points per unit volume) from Stoyan et al. (1987):

$$
\begin{aligned}
\rho^{2} G(r) d V_{1} d V_{2}= & P\{\text { two points of the process are } \\
& \text { located in the volume elements } \\
& \mathrm{d} V_{1} \text { and } \mathrm{d} V_{2} \text { centered in two } \\
& \text { points at the distance } r\}
\end{aligned}
$$

For a stationary point process, when $r \rightarrow \infty$ we get $G(r) \rightarrow 1$.

We can derive the pair correlation function $G(r, t)$ of the centres of non-overlapping convex grains $A^{\prime}$ with time decreasing sizes, in the random packing generated from the dead leaves model (in Stoyan and Schlather, 2000 , it is deduced for disks in 2D in the limit area fraction 0.25, using results of Matérn (1960)).

Theorem 4. In the time non homogeneous case for random packings, we have, using a normalization factor $k, D$ being the diameter of $A^{\prime}(0)$ in the direction of vector $h$, we get

$$
\begin{aligned}
& G(h, t)=0, \quad \text { for } h<D, \\
& k G(h, t)=2 \int_{0}^{t} \theta(u) \mathrm{d} u \int_{0}^{u} \theta(w) \mathrm{d} w . \\
& \exp \left(-\int_{0}^{u} \theta(v) \mu_{n}\left(A^{\prime}(u) \cup A^{\prime}(w)_{h}\right) \oplus \check{A}^{\prime}(v) \mathrm{d} v\right) \\
& \text { for } h \geq D .
\end{aligned}
$$

Since for $|h| \rightarrow \infty$ we have $G(h, t) \rightarrow 1$, we obtain for $h \geq D$

$$
\begin{gathered}
G(h, t)=\left[\int_{0}^{t} \theta(u) \mathrm{d} u \int_{0}^{u} \theta(w) \mathrm{d} w\right. \\
\left.\exp \left(-\int_{0}^{u} \theta(v) \mu_{n}\left(A^{\prime}(u) \cup A^{\prime}(w)_{h}\right) \oplus \check{A}^{\prime}(v)\right) \mathrm{d} v\right] / \\
{\left[\int_{0}^{t} \theta(u) \mathrm{d} u \int_{0}^{u} \theta(w) \mathrm{d} w\right.} \\
\exp \left(-\int_{0}^{u} \theta(v)\left(\mu_{n}\left(A^{\prime}(u) \oplus \check{A}^{\prime}(v)\right)+\right.\right. \\
\left.\left.\left.+\mu_{n}\left(A^{\prime}(w) \oplus \check{A}^{\prime}(v)\right)\right)\right) \mathrm{d} v\right]
\end{gathered}
$$

Proof. Consider the last event concerning point $x$ or point $x+h$ : at some time $u<t$, a Poisson point appeared in $\mathrm{d} V_{1}$ centered in $x$ (or in $\mathrm{d} V_{2}$ centered in $x+h)$, while at some time $w<u$ a Poisson point appeared in $\mathrm{d} V_{2}$ centered in $x+h$ (or in $\mathrm{d} V_{1}$ centered in $x), A^{\prime}(u) \cup A^{\prime}(w)_{h}$ being outside of the Boolean model generated by grains $A^{\prime}(v)$ appearing from 0 to $u$. From the theoretical expression (11), it is clear that the shape of $G(h, t)$ depends on the choice of functions $\theta(t)$ and $\lambda(t)$, but we have $G(h, t)=0$ for $h<D$ and $G(h, t)=1$ for $h \geq 2 D$.

Corollary 1. In the case of the time homogeneous model and with a fixed grain, we get, using a normalization factor $k, D$ being the diameter of $A^{\prime}$ in the direction of vector $h$, and with $A_{2}^{\prime}=A^{\prime} \oplus \check{A}^{\prime}$

$$
\begin{aligned}
& G(h, t)=0 \quad \text { for } h<D, \\
& k G(h, t)=\frac{2}{\left(\mu_{n}\left(A_{2}^{\prime} \cup A_{2 h}^{\prime}\right)\right)^{2}} . \\
& {\left[1-\exp \left(-\theta t \mu_{n}\left(A_{2}^{\prime} \cup A_{2 h}^{\prime}\right)\right)\left(1+\theta t \mu_{n}\left(A_{2}^{\prime} \cup A_{2 h}^{\prime}\right)\right)\right]} \\
& \quad \text { for } h \geq D .
\end{aligned}
$$

Since for $|h| \rightarrow \infty$ we have $G(h, t) \rightarrow 1$, we obtain for $h \geq D$

$$
\begin{aligned}
& G(h, t)=\frac{\left(2^{n+1} \mu_{n}\left(A^{\prime}\right)\right)^{2}}{\left(\mu_{n}\left(A_{2}^{\prime} \cup A_{2 h}^{\prime}\right)\right)^{2}} . \\
& \frac{1-\exp \left(-\theta t \mu_{n}\left(A_{2}^{\prime} \cup A_{2 h}^{\prime}\right)\right)\left(1+\theta t \mu_{n}\left(A_{2}^{\prime} \cup A_{2 h}^{\prime}\right)\right)}{1-\exp \left(-\theta t 2^{n+1} \mu_{n}\left(A^{\prime}\right)\right)\left(1+\theta t 2^{n+1} \mu_{n}\left(A^{\prime}\right)\right)} .
\end{aligned}
$$

Eq. 12 can be written in a more condensed way. We put $q=\exp \left(-\theta t 2^{n} \mu_{n}\left(A^{\prime}\right)\right)$ and therefore we have $p(t)=\frac{1}{2^{n}}(1-q)(q$ is the probability for a point to be outside of the grains of the Boolean model with grains $A_{2}^{\prime}$, generated from 0 to $t$ ). We use

$$
\begin{aligned}
K_{2}(h) & =\mu_{n}\left(A_{2}^{\prime} \cap A_{2 h}^{\prime}\right), \\
r_{2}(h) & =K_{2}(h) / K_{2}(0) .
\end{aligned}
$$


We get

$$
\begin{aligned}
& G(h)= \text { for } h<D \\
& G(h)=\left(\frac{2}{2-r_{2}(h)}\right)^{2} . \\
& \quad \frac{1-q^{2-r_{2}(h)}\left(1-\log (q)\left(2-r_{2}(h)\right)\right)}{1-q^{2}(1-\log (q)}, \\
& \text { for } h \geq D .
\end{aligned}
$$

The limiting case is obtained for $p=1 / 2^{n}$ (or equivalently $q=0$ and $t \rightarrow \infty$ ):

$$
G(h)= \begin{cases}0 & \text { for } h<D, \\ \left(\frac{2}{2-r_{2}(h)}\right)^{2} & \text { for } h \geq D .\end{cases}
$$

In $\mathbb{R}$, if $A^{\prime}$ is the segment with length 1 , we have $r_{2}(h)=1-\frac{h}{2}$ for $h \leq 2$. It comes

$$
\begin{aligned}
& G(h)=0 \quad \text { for } h<1, \\
& G(h)=\left(\frac{2}{1+h / 2}\right)^{2} \frac{1-q^{1+h / 2}(1-\log (q)(1+h / 2))}{1-q^{2}(1-\log (q)} \\
& \quad \text { for } 1<h<2, \\
& G(h)=1 \quad \text { for } h \geq 2 .
\end{aligned}
$$

We get

$$
G(1)=\frac{16}{9} \frac{1-q^{3 / 2}\left(1-\frac{3}{2} \log (q)\right)}{1-q^{2}(1-\log (q))}
$$

so that there is a strong influence of the volume fraction on the shape of $G(r)$.

For non overlapping disks with radius $R$ in the twodimensional space, we have to use Equation (13) with $D=2 R$ and

$$
\begin{aligned}
r_{2}(h) & =\frac{K(h)}{K(0)} \\
& =\arccos \left(\frac{h}{4 R}\right)-\frac{h}{4 R} \sqrt{1-\left(\frac{h}{4 R}\right)^{2}}, \text { for } h \leq 4 R \\
r_{2}(h) & =0, \quad \text { for } h \geq 4 R .
\end{aligned}
$$

For spherical grains with radius $R$ in the threedimensionnal space, we have

$$
\begin{array}{ll}
r_{2}(h)=1-\frac{3 h}{8 R}+\frac{h^{3}}{128 R^{3}} & \text { for } h<4 R, \\
r_{2}(h)=0 & \text { for } h \geq 4 R .
\end{array}
$$

\section{CONCLUSION}

Intact grains of the dead leaves model provide random packings with a volume fraction controlled by a wise combination of the time sequence of density $\theta(t)$ and of sizes $\lambda(t)$. The strength of this approach is to provide dense random packings, as opposite to deterministic constructions, and to get access to many theoretical results, such as the time evolution of the volume fraction during the construction of the packing, the size distribution of grains, and the pair correlation function of centres of grains. Contrarily to other developped approaches, like in Delarue and Jeulin (2001) for sphere packings, or in Altendorf and Jeulin (2011) for simulations of random fiber systems, it does not require the implementation of complex translations of objects controlled by external forces. The obtained space arrangement revealed by the pair correlation function of grains involves correlations on sizes up to twice the maximum diameter of grains of the model, and does not show any long range arrangements or alignments, as could be present in periodic or in some real media.

Using similar convex grains symmetrical with respect to the origin, not limited to spheres, very dense packings can be theoretically obtained, up to a full covering of space by non-overlapping convex grains, a particular case being spherical grains. Considering convex cells delimited by the boundaries of grains, tessellations of space with compact convex cells are generated for appropriate sequences asymptotically leading to a full covering of space. Models with different grain size distributions are obtained by this process. Packings with extremal volume fractions (close to 1) involve huge ranges of sizes (the smallest diameters of grains tending to 0 ), which is theoretically feasible, but practically out of reach of computer simulations.

\section{REFERENCES}

Altendorf H, Jeulin D (2011). Random-walk-based stochastic modeling of three-dimensional fiber systems. Phys Rev E 83:041804.

Andersson J, Häggström O, Månsson M (2006). The volume fraction of a non-overlapping germ-grain model. Electron Commun Probab 11:78-88.

Aste T, Weaire D (2000). The pursuit of perfect packing. Bristol: Institute of Physics.

Delarue A, Jeulin D (2001). Multi-scale simulations of spherical aggregates. Proc 8th Eur Congr Stereol. Image Anal Stereol 20:181-6.

Hashin Z (1962). The elastic moduli of hetrogeneous materials. J Appl Mech 29:143-50. 
Jeulin D (1980). Multi-component random models for the description of complex microstructures. Proc 5th Int Congr Stereol. Mikroskopie 37:130-7.

Jeulin D (1987). Random structure analysis and modelling by mathematical morphology. In: Spencer AJM, ed. Proc 5th Int Symp Contin Model Discrete Syst. Rotterdam: Balkema. pp 217-26.

Jeulin D (1989). Morphological modeling of images by sequential random functions. Signal Process 16:403-31.

Jeulin D (1993). Random models for the morphological analysis of powders. J Microsc 172:13-21.

Jeulin D (1997). Dead leaves models: From space tesselation to random functions. In Jeulin D, ed. Proc Symp Adv Theor Appl Random Sets. Singapore: World Scientific, pp 137-56.

Jeulin D (1998). Probabilistic models of structures. In: Frantziskonis GN, ed. Proc Worksh PROBAMAT 21st Cent. NATO ASI Ser 46:233-57.
Jeulin D (2000) Random texture models for materials structures. Stat Comput 10:121-31.

Jeulin D (2002). Modelling random media. Proc 8th Eur Congr Stereol. Image Anal Stereol 21:S31-S40.

Kiderlen M, Hörig M (2013). Matérn's hard core models of types I and II with arbitrary compact grains. CSGB Res Rep No 5, July 2013.

Matérn B (1960). Spatial variation. Medd Stat Skogsforkning 49(5):1-144.

Matheron G (1968). Schéma booléen séquentiel de partition aléatoire. Paris: School of Mines.

Stoyan D, Kendall WS, Mecke J (1987). Stochastic geometry and its applications. New York: Wiley.

Stoyan D, Schlather M (2000). Random sequential adsorption: Relationship to dead leaves and characterization of variability. J Stat Phys 100:969-79. 